\title{
Investigation of High Energy Density Matter for Science-Based Stockpile Stewardship
}

Progress Report: April 1, 1998 - Sept. 31, 1998

Principal Investigator: Bruno Bauer, UNR

Coinvestigators: P.L. Altick, V.L. Kantsyrev, N. Le Galloudec, R.C. Mancini, P.A. Neill, R.A. Phaneuf, G.S. Sarkisov, A.S. Shlyaptseva, J.S. Thompson, P. Winkler, and F. Winterberg, UNR Physics; J.W. Farley, UNLV Physics

\section{Statement of Work}

Activate the Nevada Terawatt Facility (NTF). Experimentally investigate z-pinch physics, in collaboration with SNL, LANL, and LLNL. Develop $x$-ray and laser diagnostics for the NTF, SNL-Z, and LANL-ATLAS. Refine atomic and radiation physics calculations and data to meet the challenges of EUV and $x$-ray diagnostic development.

\section{Progress to Date}

\section{Set up of the first experiment}

The refurbishment and installation of Zebra, a high-repetition-rate 2-terawatt pulsed-power device (from Los Alamos National Laboratory), is almost complete. This device, the highest-power electrical generator for plasma physics research at a U.S. university, forms the core of the Nevada Terawatt Facility (NTF). The NTF laboratory has undergone substantial renovation, including the creation of a high-bay area and installation of a 7.5-ton overhead crane. Bechtel Nevada safety engineers have helped the NTF meet DOE safety standards, with appropiiate control and security systems and authorization basis documents.

A separate experiment has been set up to measure the heating and expansion of wires with current prepulses similar to those at the Sandia National Lab Z facility. Wires are exploded with a $100 \mathrm{kV}, 2 \mathrm{kA}$ pulse generator and observed with optical and laser diagnostics. They will also be observed with $x$-ray backlighting, once the NTF is operational.

\section{Development of diagnostics}

Optical, EUV, and $x$-ray diagnostics are being developed in parallel, in two laboratories. The $x$-ray diagnostics development lab contains a laser-plasma soft $x$-ray source and conventional $x$-ray machines. The optical diagnostics 


\section{DISCLAIMER}

Portions of this document may be illegible in electronic image products. Images are produced from the best available original document. 
development lab contains two powerful Nd-glass lasers (one frequency doubled to 0.53 micron light).

A new two-dimensional $\mathrm{x}$-ray imaging spectrometer has been designed and is being prototyped. The new diagnostic uses glass capillaries to multiplex a twodimensional image of the plasma into an output array of spatially-separated pixels. This array is then spectrally dispersed by a crystal or a multilayer mirror, recorded by a temporally-gated imager, and reconstituted as an image by a computer. The result is a time-gated spectrum for each image point.

A 5-channel tunable $x$-ray spectrometer ("Polychromator") with nanosecond time resolution has been procured, to observe the evolution of the z-pinch $x$-ray emission in several bands, with different polarizations, from a selection of plasma regions. A transmission grating spectrometer has been incorporated into this spectrometer.

A multihole $x$-ray pinhole camera is being constructed around a 6-strip 0.1-nsgated MCP detector from Sandia National Laboratories.

A Pulse Sciences Incorporated $80 \mathrm{~kJ}$ Marx Generator has been obtained from Lawrence Livermore National Lab. It will be used to drive a second z-pinch, to backlight the plasma with soft $\mathrm{x}$-rays, and observe the evolution of the plasma density.

Laser absorption imaging, interferometry, polarimetry, collective Thomson scattering, and induced fluorescence diagnostics are being developed. Three laser systems are being developed for these laser diagnostics. First, a large, longpulse Nd:glass laser system (a Quantel PG-48 [producing 25J in 20ns] plus additional amplifiers) from Lockheed will be used to drive collective Thomson scattering and pump a dye laser to drive laser induced fluorescence. The first stages of this laser are running; the remainder will be set up once the laser has been relocated to the NTF laser room, currently under renovation. Second, a powerful subnanosecond Continuum Nd:YLF laser (also from Lockheed) is being restored, for the imaging of laser absorption, interferometry, and Faraday rotation of polarization. Last, a dye laser has been obtained (from TRW) for laser induced fluorescence measurements. Detection systems for these diagnostics are under development. A wide variety of other optical diagnostic components have been or are being procured.

A dozen fast Tektronix digital oscilloscopes ( $500 \mathrm{MHz}$ to $1 \mathrm{GHz}$ bandwidth, 2 to $5 \mathrm{GS} / \mathrm{s}$ sample rates, 4 to 16 channels) have been procured, for diagnostics of both the pulse generator and the $z$-pinch plasma. 
Calculations are being performed to determine how to use $x$-ray polarization spectroscopy to measure the electron distribution function and the magnetic field in dense $z$-pinch plasma. X-ray polarization experiments have been performed at LLNL-EBIT to test plasma spectroscopy numerical calculations.

\section{Computer simulation}

The LANL two-dimensional radiation-MHD simulation code MHRDR is currently being ported from Cray Fortran to standard Fortran (for use on workstations). MHRDR was used to simulate Zebra at LANL by Dr. Peter Sheehey, for his PhD dissertation. Sheehey (LANL) is helping with the port and with initiating the simulations (e.g., helping to set up the correct boundary conditions for the experiment). Drs. David Reisman and Jim Hammer of LLNL have offered additional modeling support with their recently updated Trac II and Trac III codes.

\section{Conference Presentations}

B.S. Bauer, V.L. Kantsyrev, N. Le Galloudec, R.C. Mancini, G.S. Sarkisov, A.S. Shlyaptseva, F. Winterberg, UNR, J.W. Farley, UNLV, and J.S. De Groot, UC Davis, "Dense Z-Pinch Research at the Nevada Terawatt Facility," BAPS 43, 1909 (1998), 40th Annual Meeting, APS Division of Plasma Physics, New Orleans, Nov 16-20, 1998.

\section{Plans for the next 6 months \\ (Oct. 1, 1998 - March 31, 1998)}

Laser and optical measurements will be made of prepulse-driven exploding wires. Observations of heating and expansion will be compared with the modeling results of $\mathrm{D}$. Reisman et al. at LLNL.

The installation of the 2 TW z-pinch (Zebra) at the NTF will be completed. Pulse generation will be tested on dummy loads at the NTF in Jan 1999. The first zpinch plasma will be made, shortly afterwards, with a single-central-wire load. The first NTF experiment will use Zebra to simultaneously drive several $x$-pinch backlighter loads. The ongoing prepulse-driven exploding wire experiment will be moved to the NTF and backlit with $x$-pinch generated $x$-rays.

Substantial diagnostic development will continue. A prototype of the 2-dimensional $x$-ray imaging spectrometer will be developed. The laser system for laser diagnostics will be repaired and installed at the NTF. Four used streak cameras will be tested and restored. Plasma spectroscopy calculations will be improved and compared with experiments at LLNL-EBIT. 